\title{
乌s \\ Purified self-amplified spontaneous emission free-electron lasers with slippage-boosted filtering
}

\author{
Dao Xiang, Yuantao Ding, and Zhirong Huang \\ SLAC National Accelerator Laboratory, Menlo Park, California 94025, USA
}

Haixiao Deng

Shanghai Institute of Applied Physics, Chinese Academy of Sciences, Shanghai, 201800, China

(Received 9 December 2012; published 22 January 2013)

\begin{abstract}
We propose a simple method to significantly enhance the temporal coherence and spectral brightness of a self-amplified spontaneous emission (SASE) free-electron laser (FEL). In this purified SASE (pSASE) FEL, a few undulator sections (called slippage-boosted sections) resonant at a subharmonic of the FEL radiation are used in the middle stage of the exponential growth regime to amplify the radiation while simultaneously reducing the FEL bandwidth. In this slippage-boosted section, the average longitudinal velocity of electrons is reduced, which effectively increases the FEL slippage length that allows the radiation fields initially far apart to create a phase relation, leading to $n$ times increase in FEL cooperation length, where $n$ is the ratio of the resonant wavelength of the slippage-boosted section to that of the original FEL radiation. The purified radiation, as a seed with improved temporal coherence, is further amplified to saturation in the undulator sections tuned to the FEL wavelength. Using the linac coherent light source II (LCLS-II) parameters as an example, we show that with the proposed configuration the temporal coherence and spectral brightness of a SASE FEL can be significantly enhanced. This scheme may be applied to many SASE FEL light sources to enhance the FEL performance.
\end{abstract}

\section{INTRODUCTION}

High-gain free-electron lasers (FELs) working in the self-amplified spontaneous emission (SASE) mode [1,2] have been successfully operated in the x-ray wavelengths [3-5], which marked the beginning of a new era of x-ray science. Starting from shot noise in the initial beam longitudinal density distribution, the output of a SASE FEL typically has rather limited temporal coherence with a spiky spectrum. Improving the FEL temporal coherence has been a topic of recent interest, and various seeding techniques in which a coherent seed is introduced to dominate over the shot noise have been proposed to reach this goal [6-12]. Seeding with external lasers typically suffers from limited frequency up-conversion efficiency, which together with noise amplification (see, for example [13-15]) in the harmonic generation process make it difficult to reach subnanometer wavelengths. While the selfseeding technique has been demonstrated at hard x-ray wavelengths [16], it requires roughly twice the undulators to reach saturation and it appears to be a challenging task to reduce the FEL power fluctuations due to the intrinsic fluctuation of the monochromatized seed and electron beam energy jitter.

Published by the American Physical Society under the terms of the Creative Commons Attribution 3.0 License. Further distribution of this work must maintain attribution to the author(s) and the published article's title, journal citation, and DOI.
It is well known that the temporal structure of a SASE FEL output consists of many spikes with a full temporal width of about $2 \pi l_{c}$, where $l_{c}$ is the cooperation length that equals to the slippage length within one gain length [17]. The number of temporal spikes is roughly $N \approx l_{b} / 2 \pi l_{c}$, with $l_{b}$ being the full width of the electron beam. Accordingly, the spectrum of a SASE FEL is similarly noisy with $\sim N$ spikes, each having a frequency spread $c / l_{b}$ and the overall frequency spread of the FEL pulse is approximately $c / 2 \pi l_{c}$, where $c$ is the speed of light. This spiky output is a result of the fact that in an FEL the radiation only propagates through a fraction of the electron bunch such that radiation fields with distance larger than $2 \pi l_{c}$ evolve independently and therefore are uncorrelated in phase.

In this paper, we propose a simple method to increase the coherence length by speeding up the slippage in an undulator tuned to a subharmonic of the FEL radiation, i.e. $\lambda_{1 / n}=n \lambda_{0}$ with $\lambda_{0}$ being the FEL radiation wavelength and $n$ is an odd number larger than one $(n=3,5,7,9, \ldots)$. Such an undulator, called slippage-boosted section, is used to amplify the SASE radiation in the exponential growth regime while simultaneously reducing the radiation bandwidth to realize a purified SASE (pSASE) FEL. In this slippage-boosted section, the average longitudinal velocity of electrons is reduced, which effectively increases the FEL slippage length that allows the radiation fields initially far apart to create a phase relation, leading to $n$ times increase in FEL coherence length. Therefore, the number of spikes in FEL temporal profile is reduced by a factor 
of $n$, leading to significant enhancement in FEL spectral brightness.

Using the LCLS-II parameters as an example, we show that even with conservative parameter sets, the FEL bandwidth can be reduced by a factor of 5 with the proposed pSASE scheme. This method will also enable FEL single spike operation [17-19] (namely reducing the number of spikes to one) with a relatively long bunch. We believe this pSASE configuration can be used in many future SASE FEL light sources to enhance the FEL performance.

\section{METHODS}

In an FEL with planar undulator, the wavelength of the on-axis radiation that can resonantly interact with the electron beam is

$$
\lambda_{n}=\frac{1+K^{2} / 2}{2 n \gamma^{2}} \lambda_{u}
$$

where $K$ is the dimensionless undulator strength related to the undulator period $\lambda_{u}$ and undulator peak field $B$ as $K=$ $0.934 \lambda_{u}[\mathrm{~cm}] B[\mathrm{~T}], \gamma$ is the relativistic factor of the beam, and $n$ is an odd number. Given the beam and undulator parameters, an FEL may operate either in the fundamental wavelength $(n=1)$ or in the harmonic lasing mode $(n>1)$ [20-24]. In the 1D limit where the beam transverse emittance and energy spread are neglected, the ratio of the power gain length for the radiation at high harmonic $L_{1 \mathrm{D}}^{(n)}$ to that at the fundamental wavelength $L_{1 \mathrm{D}}^{(1)}$ is $[20,22,24-26]$

$$
\frac{L_{1 \mathrm{D}}^{(n)}}{L_{1 \mathrm{D}}^{(1)}}=\left(\frac{A_{1}^{2}}{n A_{n}^{2}}\right)^{1 / 3},
$$

where $A_{n}$ is the coupling factor for the $n$th harmonic,

$$
A_{n}(K)=J_{(n-1) / 2}\left(\frac{n K^{2}}{4+2 K^{2}}\right)-J_{(n+1) / 2}\left(\frac{n K^{2}}{4+2 K^{2}}\right) .
$$

The gain length ratio for various undulator strengths is shown in Fig. 1, where one can clearly see that the ratio is always larger than 1 . Because the interaction between radiation and electron beam is most efficient for $n=1$,

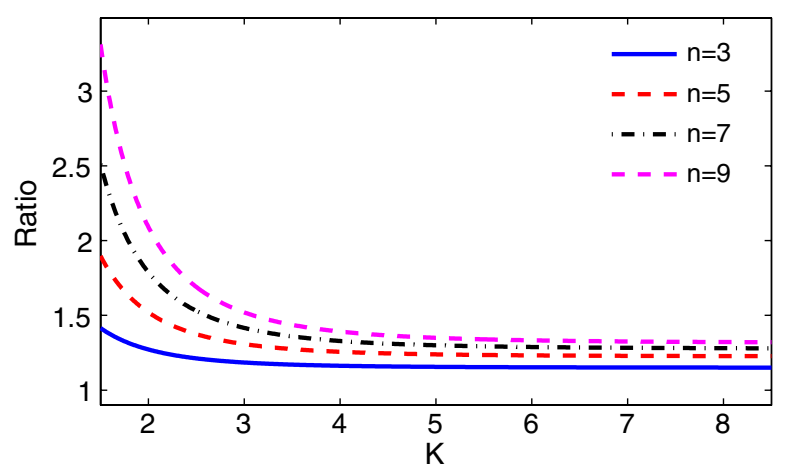

FIG. 1. Ratio of power gain length $L_{1 \mathrm{D}}^{(n)} / L_{1 \mathrm{D}}^{(1)}$ for various undulator $K$ values. most of the FELs work in the fundamental mode. To access shorter radiation wavelength with a beam limited in energy, an FEL may alternatively operate in the harmonic lasing mode, for which case the suppression of the growth of the fundamental radiation is required. This can be achieved by using phase shifters (e.g. minichicanes) to disrupt the interaction between the electron beam and the fundamental radiation while maintaining the interaction between the electron beam and the harmonic radiation field. For instance, the growth of the radiation power at the fundamental wavelength $\lambda_{1}$ can be suppressed by shifting the radiation by $\lambda_{1} / 3$ after each undulator section. In contrast, the growth of the radiation power at the 3rd harmonic is unhindered, for the phase shift is just $2 \pi$ [20,24].

While the main purpose of harmonic lasing is to extend the FEL operation to a shorter wavelength regime, it has also been realized that harmonic lasing also increases the FEL coherence length [27], compared to the case when undulator $K$ value is retuned to produce an FEL pulse having the same wavelength with fundamental lasing mode, because the total slippage length is $n$ times longer. However, in order to let the $n$th harmonic lase to saturation, all the interactions at longer wavelengths need to be suppressed, because their gain lengths are shorter, as can be seen in Fig. 1. For instance, to achieve lasing at the 7 th harmonic, one has to suppress the lasing at the fundamental, 3rd and 5th harmonics, which might make harmonic lasing at very high harmonics difficult to implement. Furthermore, the saturation power of an FEL operating in the harmonic lasing mode is lower than the nominal case when the undulator is retuned to provide the same wavelength in the fundamental lasing mode [20,22]. It is worth mentioning that one may also use minichicanes (if available) between undulator sections to shift the radiation field forward to increase the slippage length, which will also improve the SASE FEL temporal coherence [28-30].

In this paper, we study a new configuration in which neither the phase shifter to disrupt the lasing at the fundamental and lower harmonics nor the minichicanes to shift the radiation forward with respect to the electron beam is needed, yet it still provides an FEL pulse with a significantly purified spectrum, compared to the FEL working in the standard SASE mode.

The schematic layout of this pSASE FEL is illustrated in Fig. 2. The proposed pSASE FEL consists of three undulator sections, U1, U2, and U3. The first undulator section $\mathrm{U} 1$, resonant at the target FEL wavelength $\left(\lambda_{1}=\lambda_{0}\right)$, is

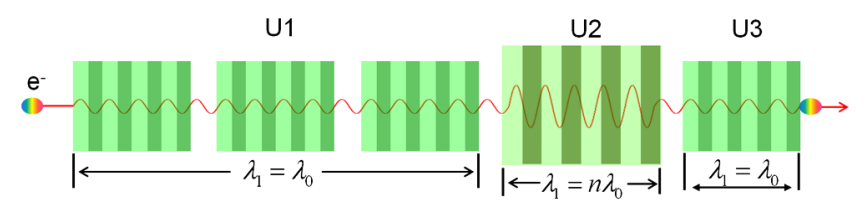

FIG. 2. Schematic layout of a pSASE FEL. 
used to produce a standard SASE radiation pulse with central wavelength at $\lambda_{0}$. The length of $\mathrm{U} 1$ is chosen in such a way that the FEL process is in the middle stage of the exponential growth regime, where the power of the SASE radiation is much higher than the spontaneous radiation while the energy spread growth from FEL interaction is negligible. The SASE radiation and the electron beam then enters the second undulator section $\mathrm{U} 2$ which is resonant at the subharmonic of the target FEL wavelength (by increasing $\lambda_{u}$ and/or $K$ ), i.e. $\lambda_{1}=n \lambda_{0}$ and $\lambda_{n}=\lambda_{0}$. In U2 (called slippage-boosted section) the SASE radiation is amplified through the harmonic interaction with its bandwidth simultaneously reduced. This is because the average longitudinal velocity of electrons $\left(v_{z} / c=1-\lambda_{1} / \lambda_{u}\right)$ in U2 is reduced, which effectively increases the FEL slippage length that allows the radiation fields initially far apart to create phase relation, leading to $n$ times increase in FEL cooperation length. The purified radiation is then further amplified to saturation in the last undulator section U3 which is again resonant at the FEL radiation wavelength $\left(\lambda_{1}=\lambda_{0}\right)$. With this configuration a pSASE FEL reaches saturation at a similar power level as that of a standard SASE FEL, with significantly enhanced temporal coherence and spectral brightness.

The length of U2 should be properly chosen to make sure the FEL power at the fundamental wavelength $\lambda_{1}=$ $n \lambda_{0}$ is much smaller than that at the harmonic wavelength $\lambda_{n}=\lambda_{0}$ such that phase shifters are not needed to suppress the lasing at fundamental. This is made possible because the radiation at $n \lambda_{0}$ starts from shot noise while the radiation at $\lambda_{0}$ is seeded by the radiation produced in $\mathrm{U} 1$. Typically after a few gain lengths, the growth rate of the FEL power at $\lambda_{0}$ slows down in $\mathrm{U} 2$, because the gain length at the harmonic wavelength is more sensitive to energy spread growth.

Take the LCLS-II under construction at SLAC as an example; we assume the beam peak current is $2.5 \mathrm{kA}$, normalized transverse emittance is $0.6 \mu \mathrm{m}$, and rms energy spread is $1 \mathrm{MeV}$. LCLS-II uses a variable-gap undulator of which the undulator period length is $\lambda_{u}=5.5 \mathrm{~cm}$ and the $K$ value can be tuned from about 1 up to about 10 [31]. Assuming the beam energy is $6 \mathrm{GeV}$ and the FEL target wavelength is $\lambda_{0}=0.6 \mathrm{~nm}$, there are several options to produce intense radiation at $0.6 \mathrm{~nm}$, i.e., through fundamental lasing with $K=2\left(\lambda_{1}=0.6 \mathrm{~nm}\right), 3 \mathrm{rd}$ harmonic lasing with $K=4\left(\lambda_{3}=0.6 \mathrm{~nm}\right)$, 5th harmonic lasing with $K=5.29\left(\lambda_{5}=0.6 \mathrm{~nm}\right)$, 7th harmonic lasing with $K=6.32\left(\lambda_{7}=0.6 \mathrm{~nm}\right), \ldots$, up to 17 th harmonic lasing with $K=10\left(\lambda_{17}=0.6 \mathrm{~nm}\right)$. The 3D gain lengths (assuming average beta function of $10 \mathrm{~m}$ ) at $0.6 \mathrm{~nm}$ with various lasing scenarios for various beam energy spreads found by Xie's formula [24,32] are shown in Fig. 3.

In the cold beam limit where beam energy spread is negligible, the harmonic lasing mode is more efficient than the fundamental lasing. This is because the effective

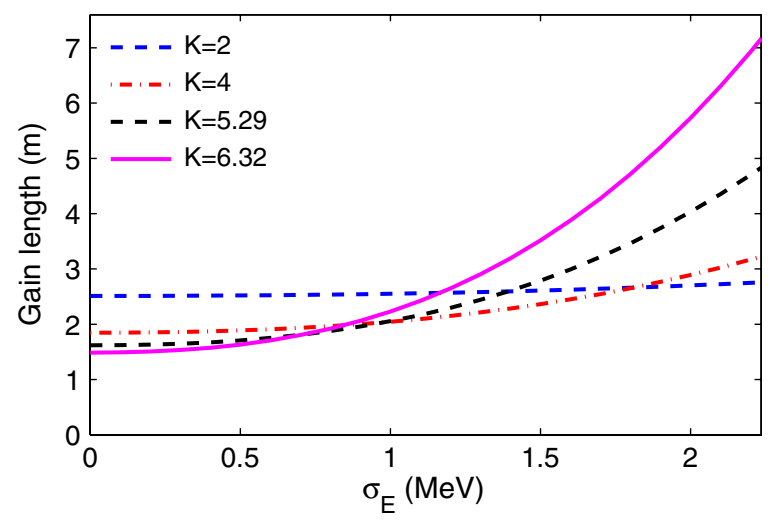

FIG. 3. Power gain length at $\lambda_{0}=0.6 \mathrm{~nm}$ as a function of beam energy spread with LCLS-II parameters for various lasing scenarios: fundamental lasing with $K=2$, 3rd harmonic lasing with $K=4$, 5th harmonic lasing with $K=5.29$, and 7th harmonic lasing with $K=6.32$.

momentum compaction of the undulator $\left(R_{56}=2 N_{u} \lambda_{1}\right.$, where $N_{u}$ is the number of periods of the undulator) is larger for harmonic lasing, which speeds up the microbunching, similar to the distributed optical klystron technique (see, for example [33]). However, as the beam energy spread increases, the debunching effect from the larger momentum compaction starts to degrade the FEL gain. As a result, the power gain length quickly grows for FEL operating in the harmonic lasing mode as the beam energy spread increases, as shown in Fig. 3. This also leads to reduced saturation power for the FEL working in the harmonic lasing mode, since the FEL power growth stops at smaller energy spread [22]. To maintain the same saturation power while reducing the FEL bandwidth, in the proposed pSASE scheme, the length of $\mathrm{U} 2$ is relatively short so that the energy spread growth is not significantly increased. The main purpose of U2 is to purify the SASE radiation generated in U1 to prepare a seed with improved temporal coherence for further amplification in U3. Operating U3 at the same wavelength as U1 makes the saturation power of a SASE FEL essentially the same as the standard SASE configuration, which allows one to increase the spectral brightness of the FEL radiation.

\section{SIMULATION}

In this section we present simulation results for a typical pSASE FEL to support our analysis in the section above. In our simulation we assume a flattop beam with peak current of $2.5 \mathrm{kA}$, full width of $40 \mathrm{fs}$ (corresponding beam charge is $100 \mathrm{pC}$ ), beam energy spread of $1 \mathrm{MeV}$, and transverse emittance of $0.6 \mu \mathrm{m}$, similar to that obtained in the startto-end simulation for LCLS-II. With a variable-gap undulator, the LCLS-II soft $x$-ray beam line will cover a broad range of $x$-ray energies from about $200 \mathrm{eV}$ to $2 \mathrm{keV}$. In the baseline design of LCLS-II [31], the soft x-ray undulators have 18 sections with a break of $1 \mathrm{~m}$ between each 

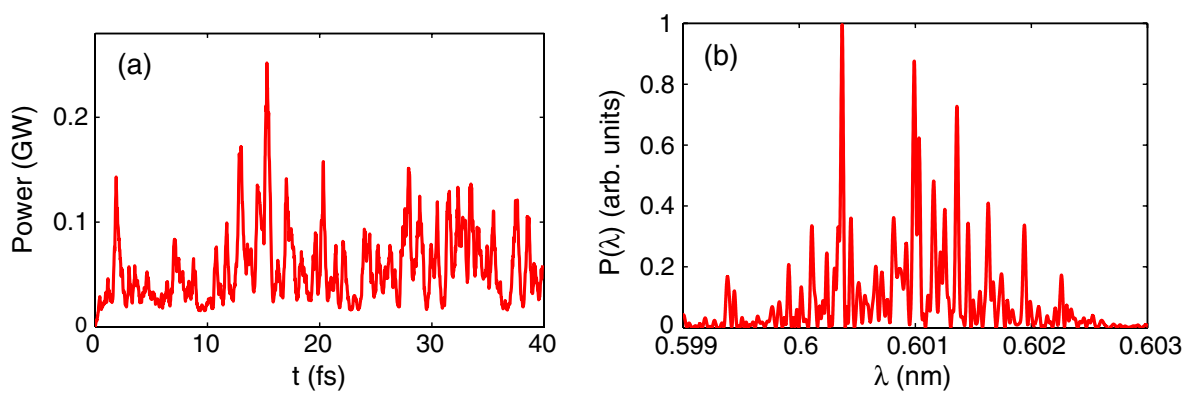

FIG. 4. FEL power (a) and spectrum (b) at the exit of U1 with nine undulator sections.

undulator. The number of periods per section is 61 and the undulator period is $5.5 \mathrm{~cm}$.

In our simulation we focus on generating $\mathrm{x}$-ray radiation at $0.6 \mathrm{~nm}$ in the LCLS-II soft $\mathrm{x}$-ray beam line using a $6 \mathrm{GeV}$ beam. Following Fig. 2, in our study the first undulator section $\mathrm{U} 1$ consists of nine undulators with the fundamental wavelength of the SASE radiation at $\lambda_{1}=$ $0.6 \mathrm{~nm}$ (corresponding $K$ value is 2). The SASE radiation generated in U1 is purified in U2 which consists of three undulators resonant at $\lambda_{1}=4.2 \mathrm{~nm}$. The corresponding undulator strength for $\mathrm{U} 2$ is $K=6.32$, which can be readily achieved by reducing the gap of the undulator. The SASE radiation is purified and amplified in U2 through the seventh harmonic interaction. Finally, the radiation with improved temporal coherence is further amplified to saturation in U3 which consists of six undulators resonant again at $\lambda_{1}=0.6 \mathrm{~nm}(K=2)$.

After nine undulator sections, the FEL power and spectrum obtained with GENESIS code [34] at the exit of U1 are shown in Fig. 4. The average power of the radiation is about $60 \mathrm{MW}$ and the energy spread growth from FEL interaction is negligible. The radiation power profile consists of $\sim 40$ spikes, each having a full width of about $1 \mathrm{fs}$.
The radiation field and particle distribution are first dumped at the exit of $\mathrm{U} 1$, and then further imported in GENESIS code for simulation of the 7th harmonic interaction in $\mathrm{U} 2$ which is resonant at $4.2 \mathrm{~nm}$. The evolution of the radiation power profile and spectrum in $\mathrm{U} 2$ are shown in Fig. 5, where one can clearly see that the radiation bandwidth is gradually reduced in U2 through the slippageboosted filtering effect. After three undulator sections, the number of spikes in the radiation power profile is reduced to $\sim 10$ [Fig. 5(e)], and the bandwidth is accordingly reduced by about a factor of 4 [Fig. 5(f)]. Further sending the beam through two more undulator sections leads to a smoother temporal profile with only seven spikes [Fig. 5(g)], but the spectrum [Fig. 5(h)] is quite similar to that after just three undulators [Fig. 5(f)]. So in our simulation, U2 only has three undulators resonant at $4.2 \mathrm{~nm}$.

Note, since U2 is relatively short, and the harmonic radiation is seeded by that produced in $\mathrm{U} 1$, it is not necessary to use phase shifters to suppress the growth of the fundamental radiation at $4.2 \mathrm{~nm}$. In our simulation, the average power of the $0.6 \mathrm{~nm}$ radiation at the exit of $\mathrm{U} 2$ is about $300 \mathrm{MW}$ while that of the fundamental radiation $(4.2 \mathrm{~nm})$ at the exit of $\mathrm{U} 2$ is only about $1 \mathrm{MW}$.
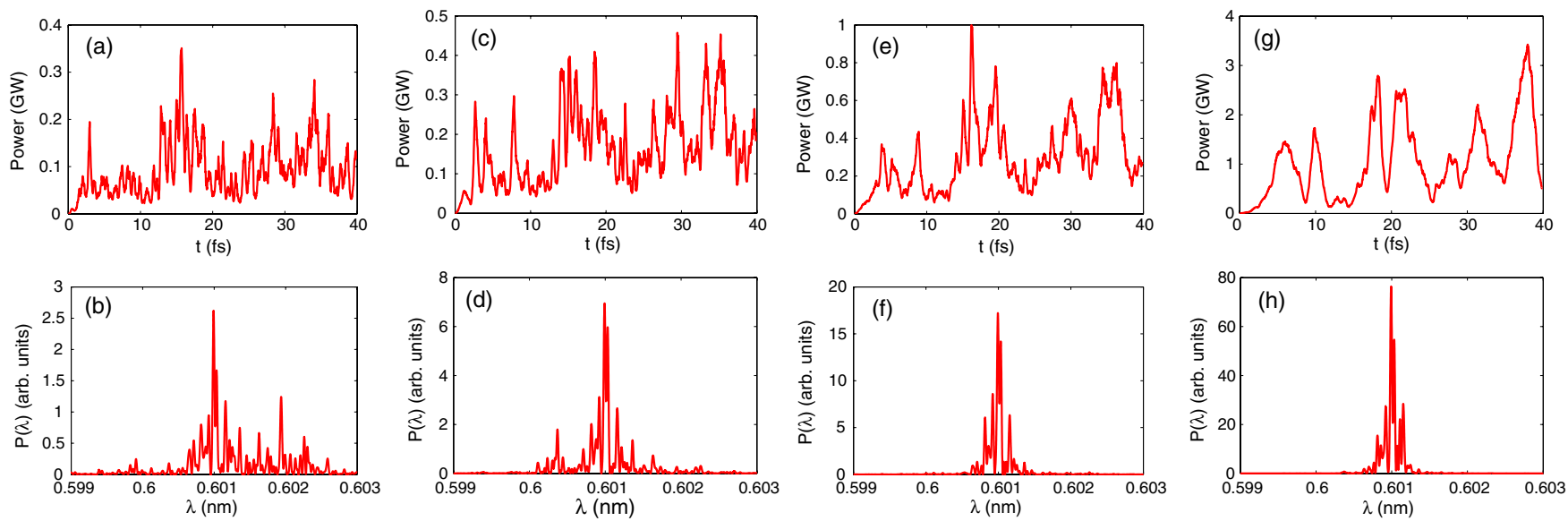

FIG. 5. SASE radiation power and spectrum at the exit of the 1st undulator in U2 [(a) and (b)]; at the exit of the 2nd undulator in U2 [(c) and (d)]; at the exit of the 3rd undulator in U2 [(e) and (f)]; at the exit of the 5th undulator in U2 [(g) and (h)]. The spectrum brightness $P(\lambda)$ is normalized to the radiation peak spectral brightness at the exit of U1 [Fig. 4(b)]. 

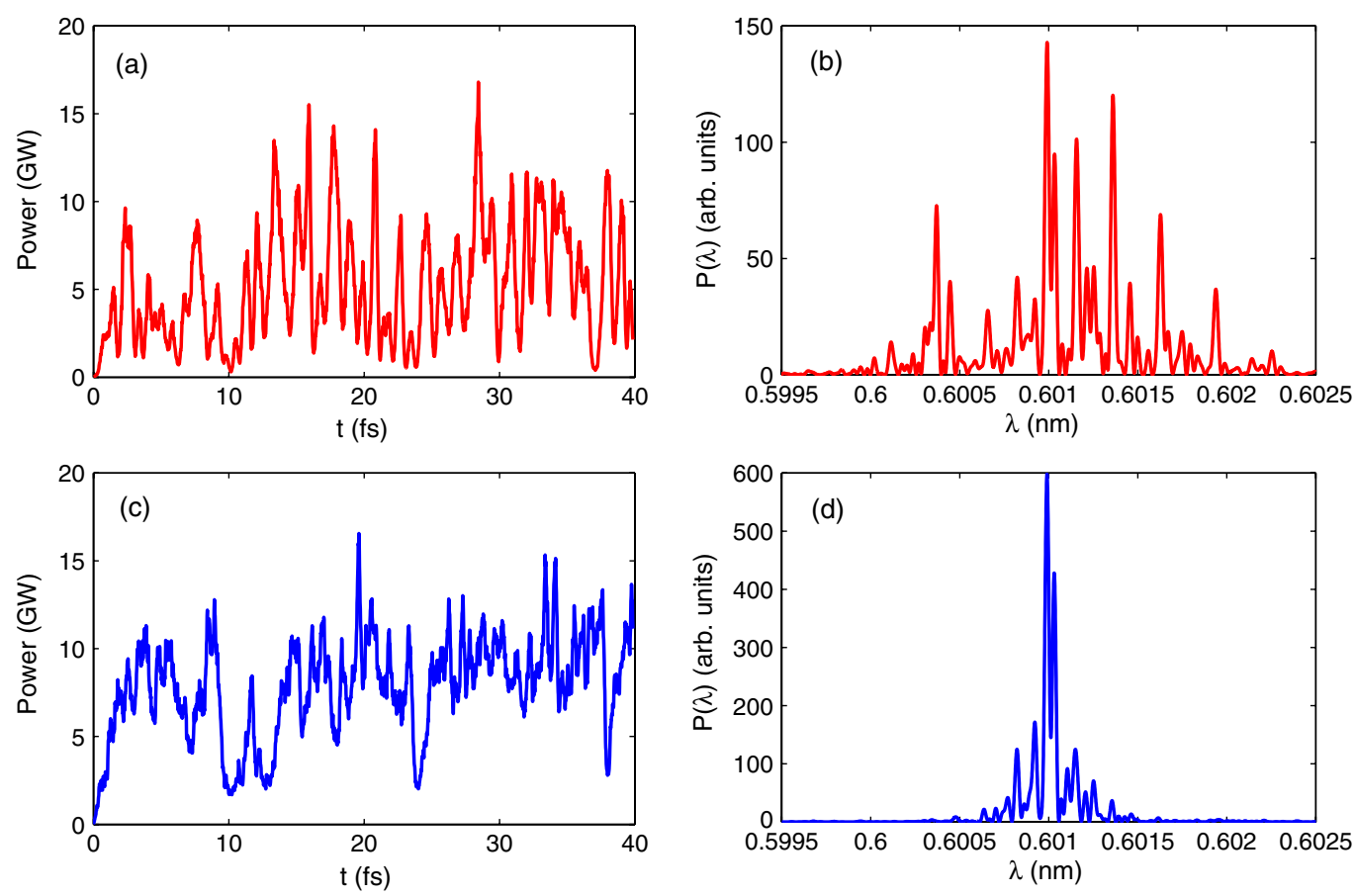

FIG. 6. Representative radiation power profiles and spectra for a standard SASE FEL [(a) and (b)] and a pSASE FEL [(c) and (d)]. The average power for both cases is about $7 \mathrm{GW}$ and in the simulation the beam parameters, lattice functions, and initial shot noise are all the same. The spectral brightness is normalized to the peak spectral brightness at the exit of U1.

Once again the radiation field and particle distribution are dumped at the exit of $\mathrm{U} 2$, and finally they are imported for simulation in $\mathrm{U} 3$ which is resonant at $0.6 \mathrm{~nm}$. With the FEL interaction in U2, the beam energy spread is increased to about $1.3 \mathrm{MeV}$ at the exit of U2. As can be seen in Fig. 3, at this energy spread level the power gain length of the 7th harmonic lasing mode exceeds that of the fundamental lasing mode. By sending the purified radiation and electron beam to U3 that operates in fundamental lasing mode, the efficiency of the FEL interaction is maximized and the saturation power for this pSASE FEL will be similar to that in a standard SASE FEL.

The purified radiation is amplified to saturation in U3 after six undulators. The radiation power profiles and spectra for an FEL working in the standard SASE mode and pSASE mode are shown in Fig. 6. For a fair comparison, the same beam parameters and initial shot noise are used in the simulation. The only difference is that for the pSASE FEL, the undulator $K$ value for the 10th, 11th, and 12 th undulators are set at $K \approx 6.32$. The average power for both modes is similar, but the bandwidth of the pSASE FEL is significantly smaller than the standard SASE FEL.

Note, the bandwidth reduction factor in a pSASE FEL is approximately $n L_{3 \mathrm{D}}^{(n)} / L_{3 \mathrm{D}}^{(1)}$, where $n$ is the harmonic number in the slippage-boosted section, $L_{3 \mathrm{D}}^{(n)}$ and $L_{3 \mathrm{D}}^{(1)}$ are the $3 \mathrm{D}$ gain lengths of the radiation at the FEL wavelength $\lambda_{0}$ in $\mathrm{U} 2$ and $\mathrm{U} 1$, respectively. Therefore, one can either increase the harmonic number (e.g., by increasing the $K$ value and/ or $\lambda_{u}$ ) or increase the power gain length (e.g., by increasing the average beta function) in $\mathrm{U} 2$ to further reduce the FEL bandwidth.

To quantify the bandwidth reduction factor, we performed ten simulations with different initial shot noise distributions and the averaged radiation spectra are shown in Fig. 7. The relative FWHM bandwidth of the radiation
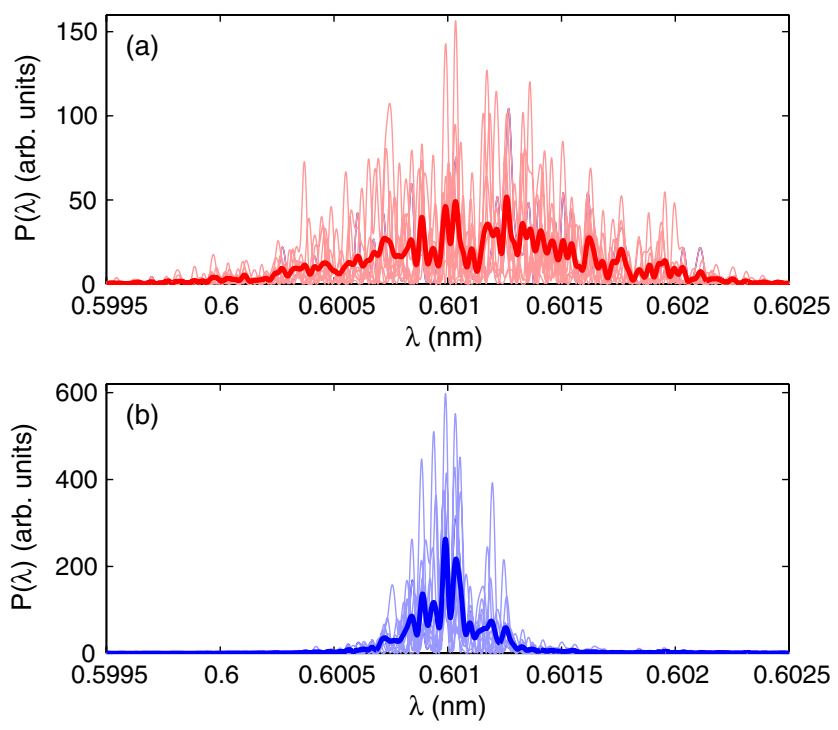

FIG. 7. Spectrum of the FEL radiation produced in the standard SASE (a) and the pSASE mode (b). Thin lines refer to single shot realizations and the bold line refers to the average over ten realizations. 
produced in the standard SASE mode is about $1.5 \times 10^{-3}$, while that produced in pSASE mode is about $3 \times 10^{-4}$. The bandwidth is reduced by approximately a factor of 5 , in good agreement with the theory $\left(n L_{3 \mathrm{D}}^{(n)} / L_{3 \mathrm{D}}^{(1)} \approx 6\right.$ for our simulation parameters).

It should be pointed out that in our simulation we conservatively chose $K=6.32$ in the slippage-boosted section. Actually for the LCLS-II undulator, the undulator strength can be tuned up to about $K=10$, which in principle allows one to use the 17th harmonic interaction in U2 to further reduce the FEL bandwidth. However, at such a high harmonic number, the FEL performance may be more sensitive to field errors, beam energy spread, etc. It is worth mentioning that reducing the FEL bandwidth also increases the taper efficiency of a saturated FEL [35]. Since the number of longitudinal modes in a pSASE FEL is reduced, one can eventually extract more power from the electron beam in a pSASE FEL compared to a standard SASE FEL. Therefore, adding tapered undulator sections to a pSASE FEL may lead to further enhancement in FEL performance.

\section{SUMMARY AND DISCUSSIONS}

We have studied a simple scheme to significantly enhance the temporal coherence and spectral brightness of a SASE FEL. In this pSASE FEL, a few undulator sections resonant at a subharmonic of the FEL radiation are used in the middle stage of the exponential growth regime to amplify the radiation while simultaneously reducing the FEL bandwidth. In this slippage-boosted section, the FEL slippage length is increased, which allows the radiation fields initially far apart to create phase relation, leading to significant increase in FEL cooperation length. The purified radiation is further amplified to saturation in the undulator sections tuned to the FEL radiation wavelength. With this configuration, a pSASE FEL reaches saturation at a similar power level as that of a standard SASE FEL, with significantly enhanced temporal coherence and spectral brightness.

Using the LCLS-II parameters as an example, we show that even with conservative parameter sets, the FEL bandwidth can be reduced by a factor of 5 with the proposed scheme. It is worth mentioning that the parameters used in our simulations and calculations are representative rather than fully optimized design sets. A more careful optimization might lead to further improvements of the scheme. Furthermore, a higher bandwidth reduction factor may be achieved with a larger $n$ and longer gain length in the slippage-boosted section. However, it is likely that the performance of a pSASE FEL with an extremely large $n$ will be more sensitive to a lot of unwanted effects and at some point the disadvantages will outweigh the benefits. These concerns will be addressed in our future work.

For SASE FELs with variable-gap undulators (such as LCLS-II and European XFEL [36]), it is straightforward to reconfigure it to pSASE mode by increasing the $K$ value of part of the undulators with no additional cost. For SASE FELs with fixed-gap undulators, since only a relatively short section (with a length comparable to $\sim 3$ gain lengths) is needed to purify the radiation spectrum, one may replace several existing undulator sections with variable-gap undulators (at a moderate cost) to enable the pSASE operation. Similarly, for SASE FELs with short-period undulators (such as SACLA [5] and SwissFEL [37]) where it may be difficult to increase the undulator $K$ value to significantly increase the slippage length in the slippageboosted section, one may replace several existing undulator sections with large-period variable-gap undulators to enable the pSASE operation. The pSASE operation mode may be particularly suited for high-repetition rate FELs where the heat load associated with the high rep-rate beam may pose potential damages to the monochromator required for self-seeding, and the pSASE scheme may be a very promising alternative for generation of radiation with narrow bandwidth. In general, this scheme may be applied to many SASE FEL light sources to enhance the FEL performance.

\section{ACKNOWLEDGMENTS}

We thank S. Reiche for useful discussions in simulation of harmonic interactions in FELs and H.-D. Nuhn for discussions of LCLS-II undulator parameters. The work of Y.D., Z.H., and D. X. was supported by the U.S. DOE under Contract No. DE-AC02-76SF00515 and the work of H. D. was supported by the Natural Science Foundation of China under Grant No. 11205234.

[1] A. Kondratenko and E. Saldin, Part. Accel. 10, 207 (1980).

[2] R. Bonifacio, C. Pellegrini, and L. M. Narducci, Opt. Commun. 50, 373 (1984).

[3] W. Ackermann et al., Nat. Photonics 1, 336 (2007).

[4] P. Emma et al., Nat. Photonics 4, 641 (2010).

[5] T. Ishikawa et al., Nat. Photonics 6, 540 (2012).

[6] L.-H. Yu, Phys. Rev. A 44, 5178 (1991).

[7] G. Stupakov, Phys. Rev. Lett. 102, 074801 (2009).

[8] D. Xiang and G. Stupakov, Phys. Rev. ST Accel. Beams 12, 030702 (2009).

[9] D. Xiang and G. Stupakov, New J. Phys. 13, 093028 (2011).

[10] J. Feldhaus, E. L. Saldin, J.R Schneider, E. A. Schneidmiller, and M. V. Yurkov, Opt. Commun. 140, 341 (1997).

[11] G. Geloni, V. Kocharyan, and E. Saldin, DESY Report No. 10-033, 2010.

[12] Y. Ding, Z. Huang, and R. Ruth, Phys. Rev. ST Accel. Beams 13, 060703 (2010).

[13] E. L. Saldin, E. A. Schneidmiller, and M. V. Yurkov, Opt. Commun. 202, 169 (2002).

[14] G. Geloni, V. Kocharyan, and E. Saldin, DESY Report No. 11-200, 2011. 
[15] D. Ratner, A. Fry, G. Stupakov, and W. White, Phys. Rev. ST Accel. Beams 15, 030702 (2012).

[16] J. Amann et al., Nat. Photonics 6, 693 (2012).

[17] R. Bonifacio, L. De Salvo, P. Pierini, N. Piovella, and C. Pellegrini, Phys. Rev. Lett. 73, 70 (1994).

[18] S. Reiche, P. Musumeci, C. Pellegrini, and J. B. Rosenzweig, Nucl. Instrum. Methods Phys. Res., Sect. A 593, 45 (2008).

[19] Y. Ding et al., Phys. Rev. Lett. 102, 254801 (2009).

[20] B. W. J. McNeil, G. R. M. Robb, M. W. Poole, and N. R. Thompson, Phys. Rev. Lett. 96, 084801 (2006).

[21] H. Deng and Z. Dai, Chinese Phys. C 32, 593 (2008).

[22] H. Deng, H. Bei, and Z. Dai, Chinese Phys. C 34, 115 (2010).

[23] J. Dai, H. Deng, and Z. Dai, Phys. Rev. Lett. 108, 034802 (2012).

[24] E. A. Schneidmiller and M. V. Yurkov, Phys. Rev. ST Accel. Beams 15, 080702 (2012).

[25] J. Murphy, C. Pellegrini, and R. Bonifacio, Opt. Commun. 53, 197 (1985).

[26] Z. Huang and K.-J. Kim, Phys. Rev. E 62, 7295 (2000).
[27] E. A. Schneidmiller and M. V. Yurkov, arXiv:1210.4677.

[28] N. R. Thompson and B. W. J. McNeil, Phys. Rev. Lett. 100, 203901 (2008).

[29] N. R. Thompson, D. J. Dunning, and B. W. J. McNeil, in Proccedings of IPAC10 (ICR, Kyoto, Japan, 2010), p. 2257.

[30] J. Wu, C. Pellegrini, and A. Marinelli (unpublished); J. Wu et al. (unpublished).

[31] LCLS-II Conceptual Design Report (unpublished).

[32] M. Xie, in Proceedings of the Particle Accelerator Conference, Dallas, TX, 1995 (IEEE, New York, 1995), p. 183.

[33] Y. Ding, P. Emma, Z. Huang, and V. Kumar, Phys. Rev. ST Accel. Beams 9, 070702 (2006).

[34] S. Reiche, Nucl. Instrum. Methods Phys. Res., Sect. A 429, 243 (1999).

[35] Y. Jiao, J. Wu, Y. Cai, A. Chao, W. Fawley, J. Frisch, Z. Huang, H.-D. Nuhn, C. Pellegrini, and S. Reiche, Phys. Rev. ST Accel. Beams 15, 050704 (2012).

[36] M. Altarelli et al., DESY Report No. 2006-097, 2007.

[37] SwissFEL Conceptual Design Report (unpublished). 